\title{
Microwave Fire Lessons Learned
}

\author{
Robert V Fox
}

December 2019

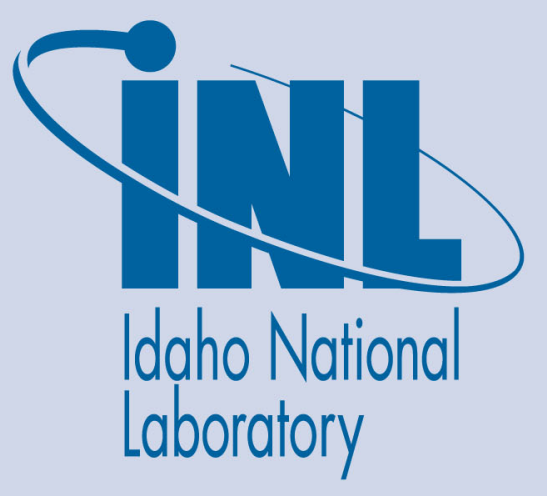

The INL is a U.S. Department of Energy National Laboratory operated by Battelle Energy Alliance 
INL/MIS-20-58098-Revision-0

\title{
Microwave Fire Lessons Learned
}

\author{
Robert V Fox
}

December 2019

\section{Idaho National Laboratory} Idaho Falls, Idaho 83415

http://www.inl.gov

Prepared for the U.S. Department of Energy Office of Nuclear Energy Under DOE Idaho Operations Office Contract DE-AC07-05ID14517 


\section{CEM Mars 6 Incident November 6, 2019}




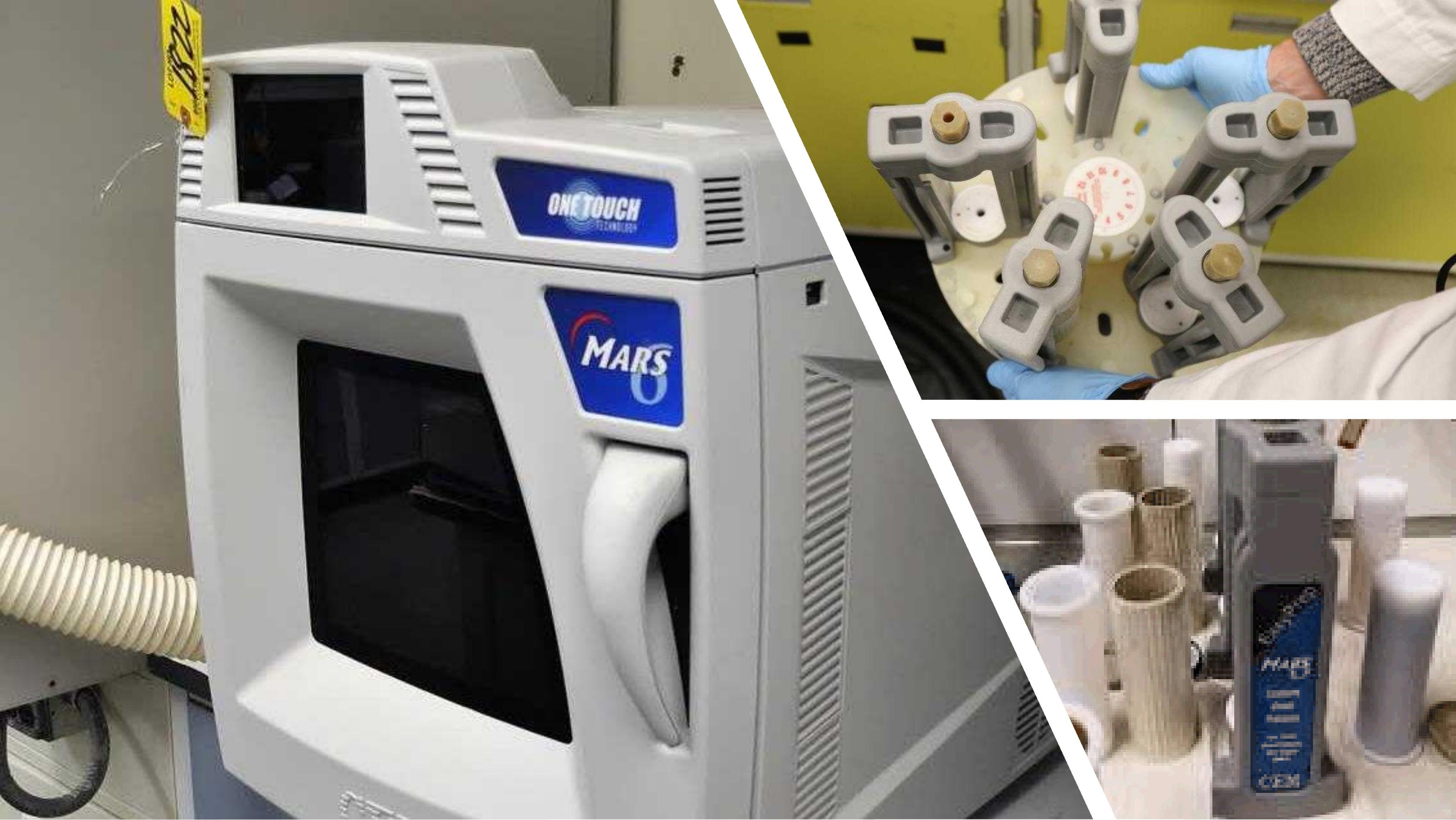




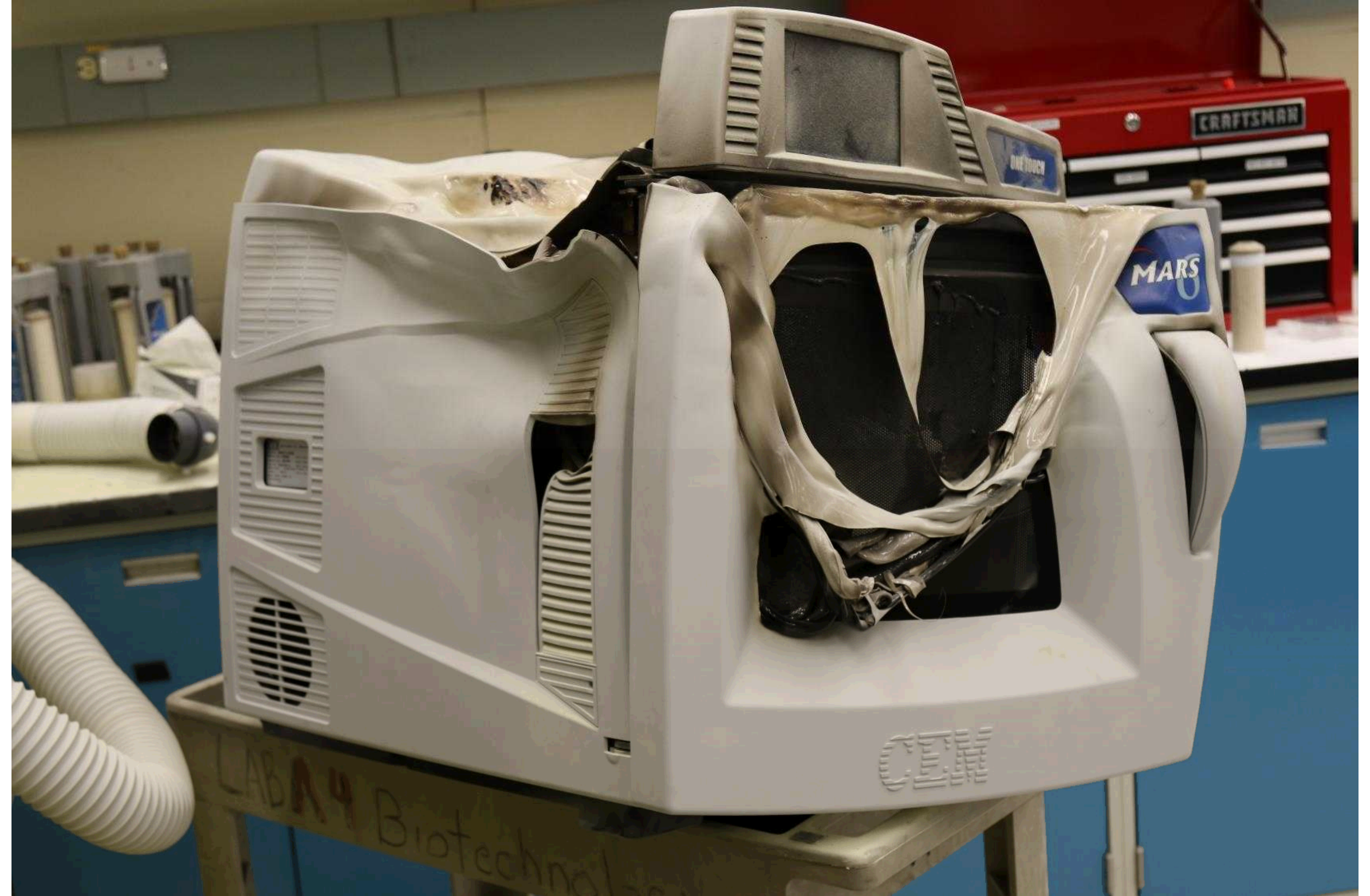




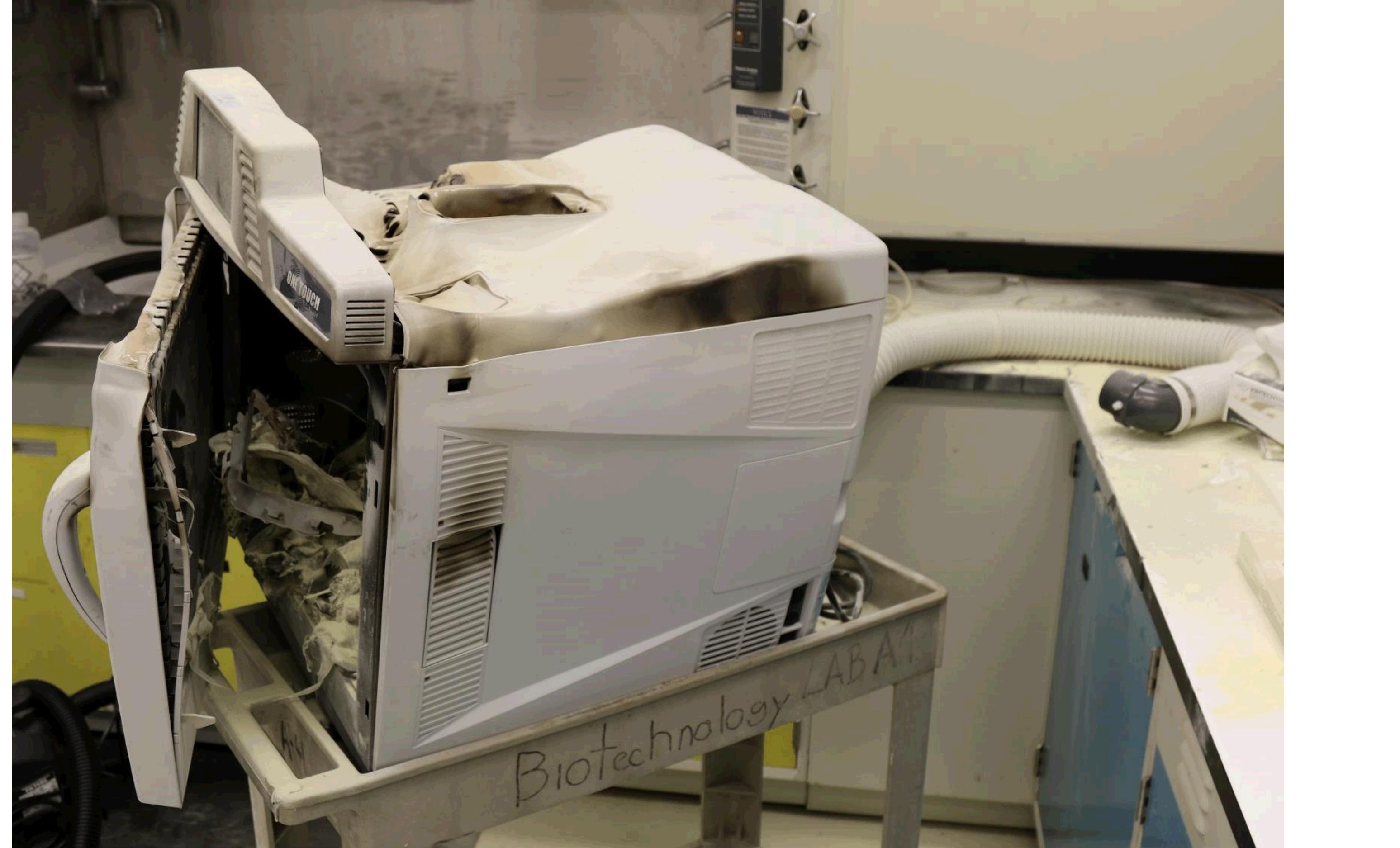




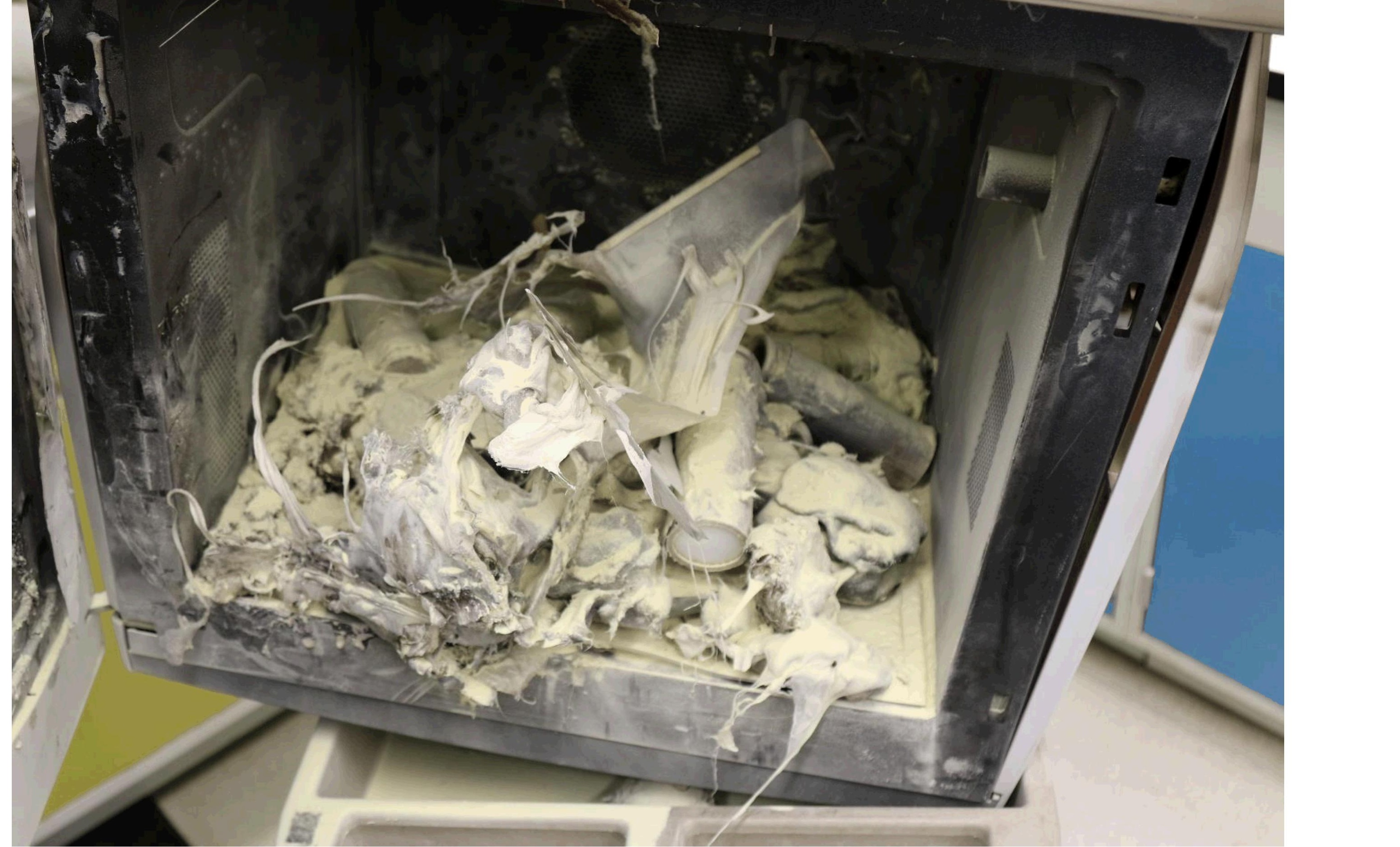




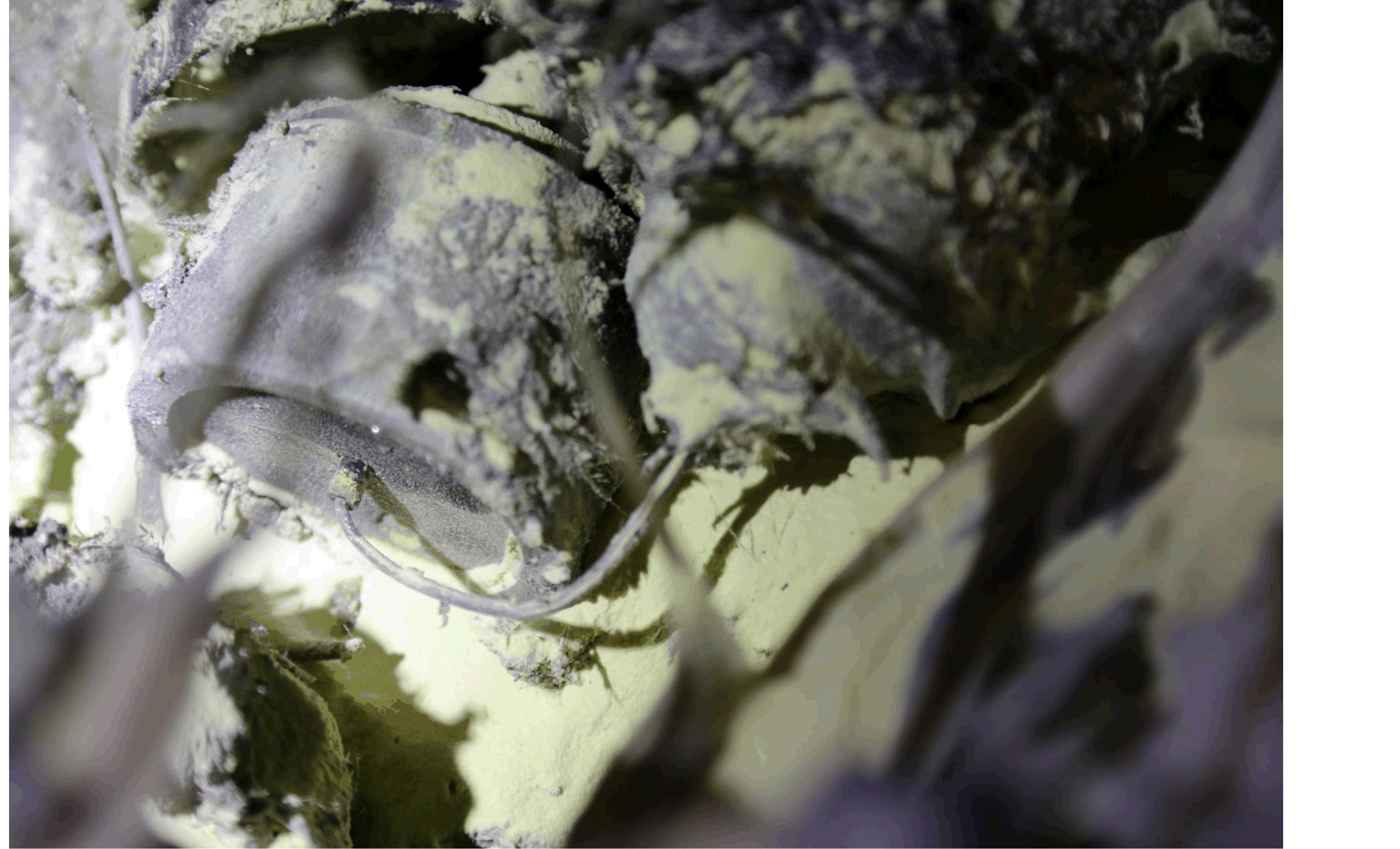




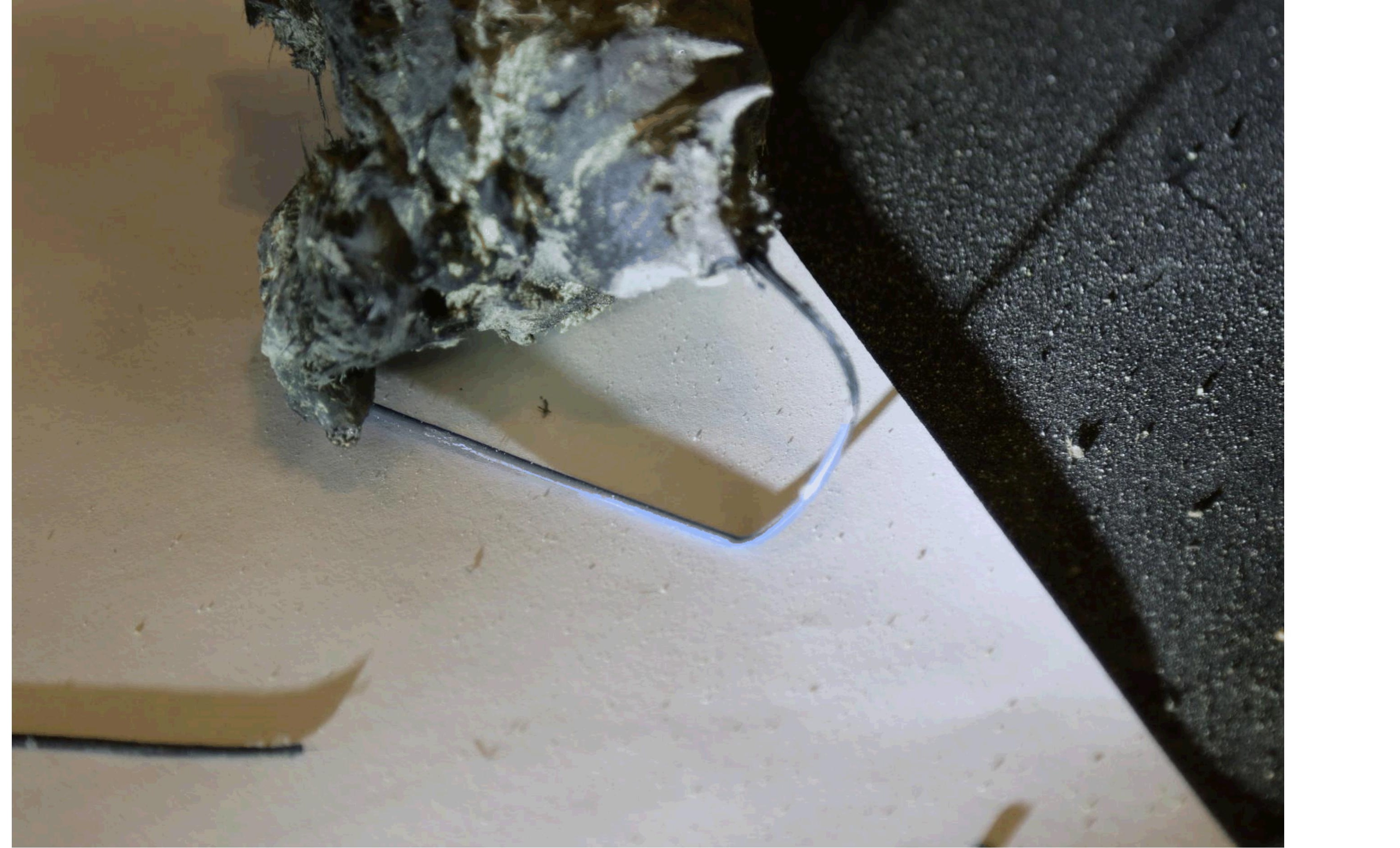




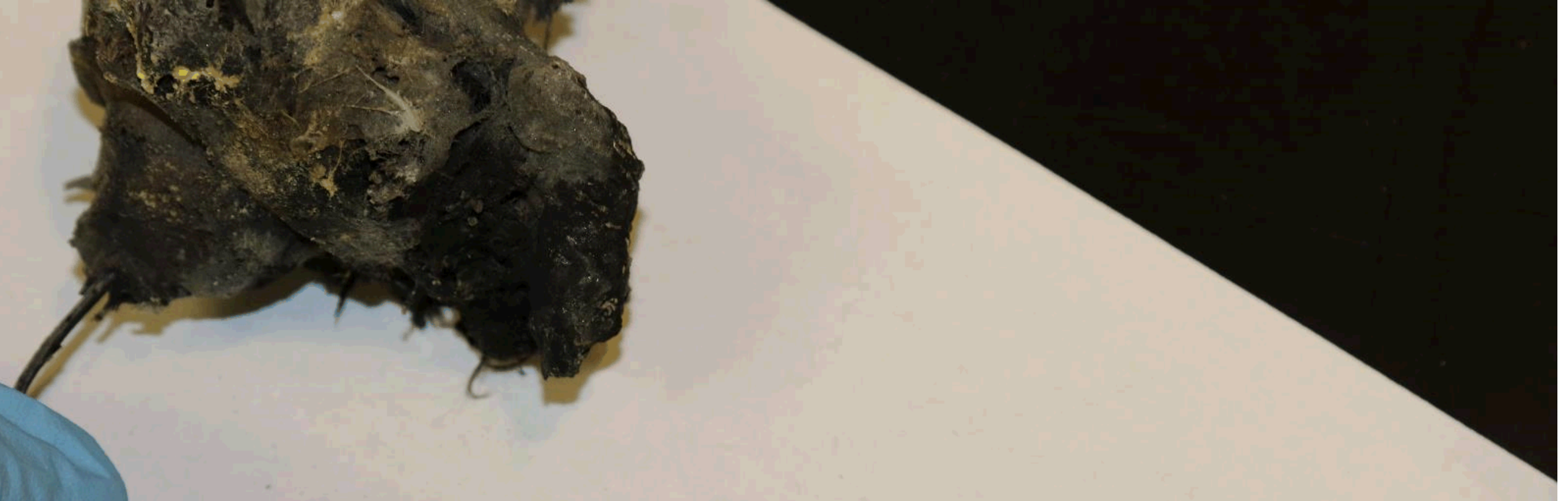

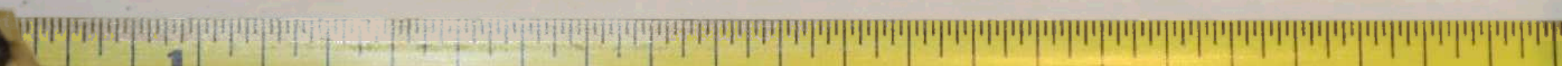

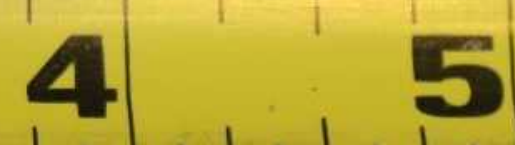

6

wir staky
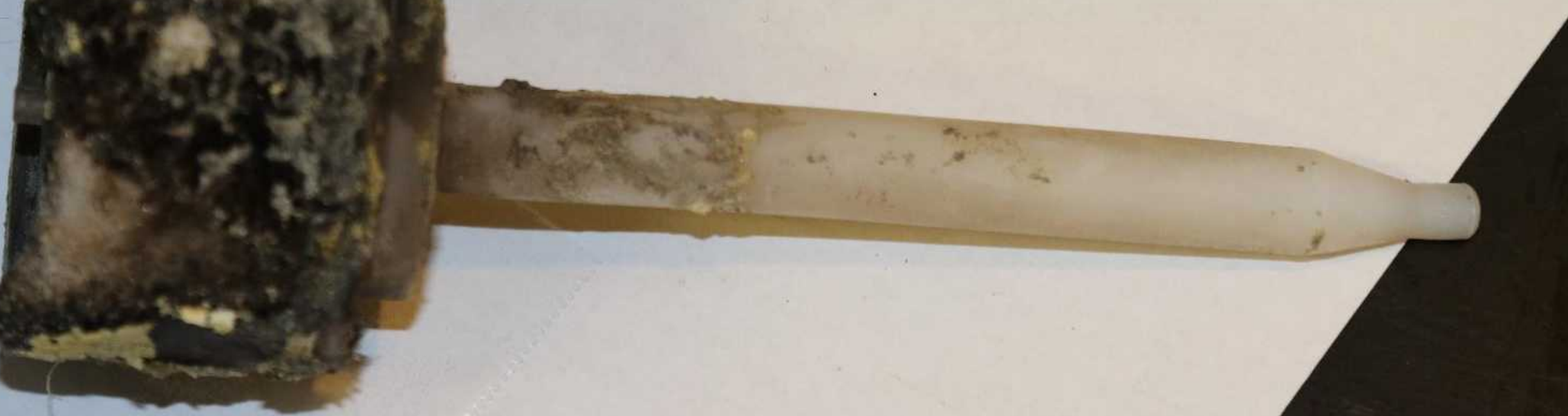


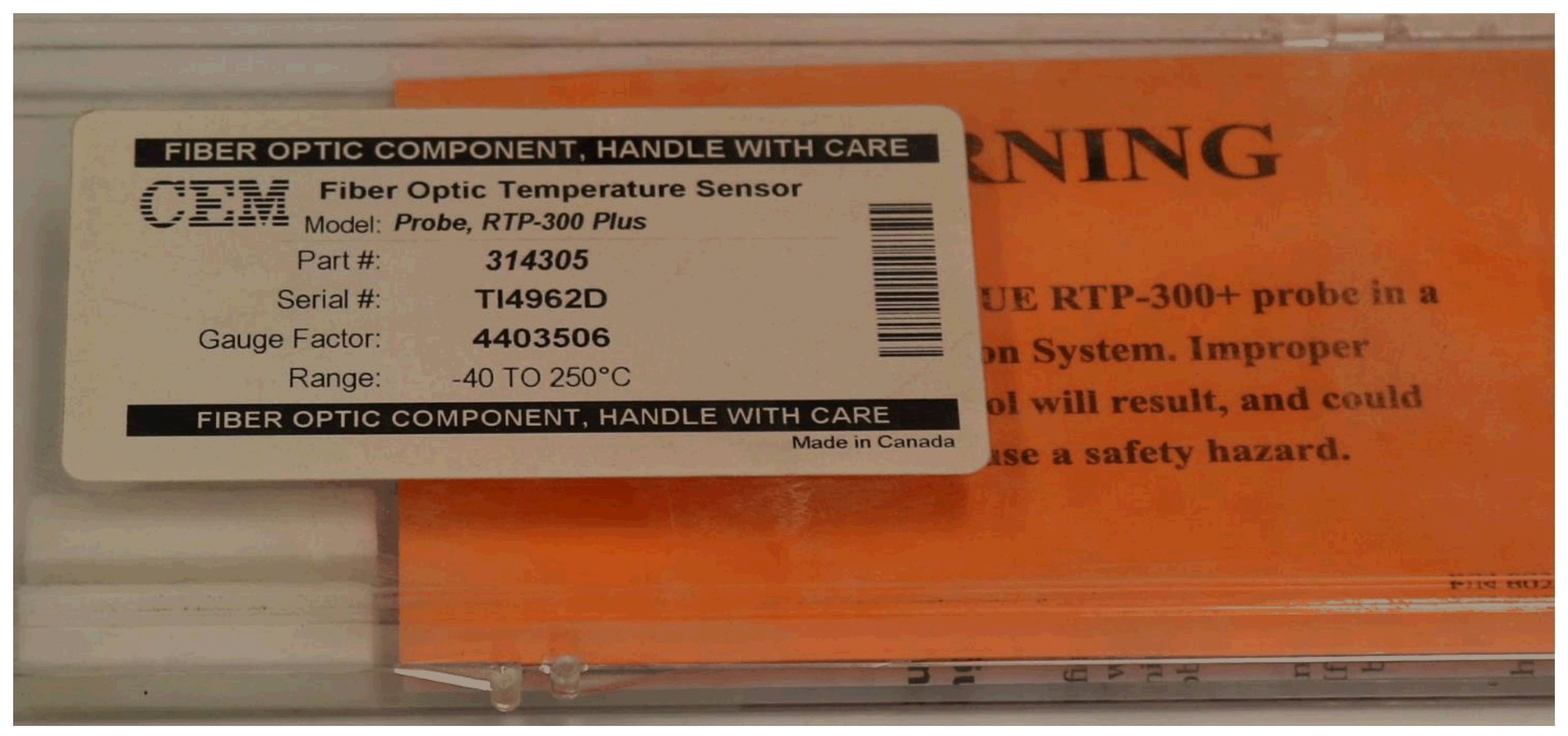


MARS 5 \& MARS $6^{\text {TM }}$

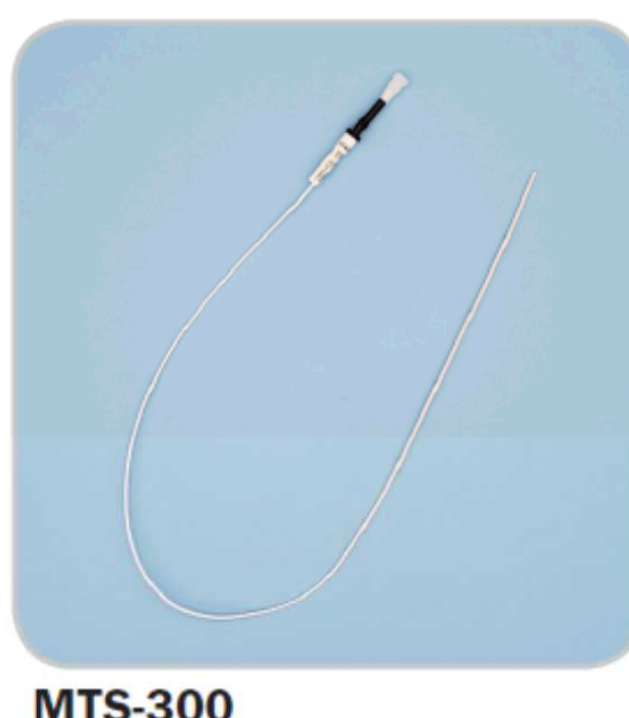

\section{P/N 314355}

White probe with white end

Temperatures $<250^{\circ} \mathrm{C}$

MARS 6 only

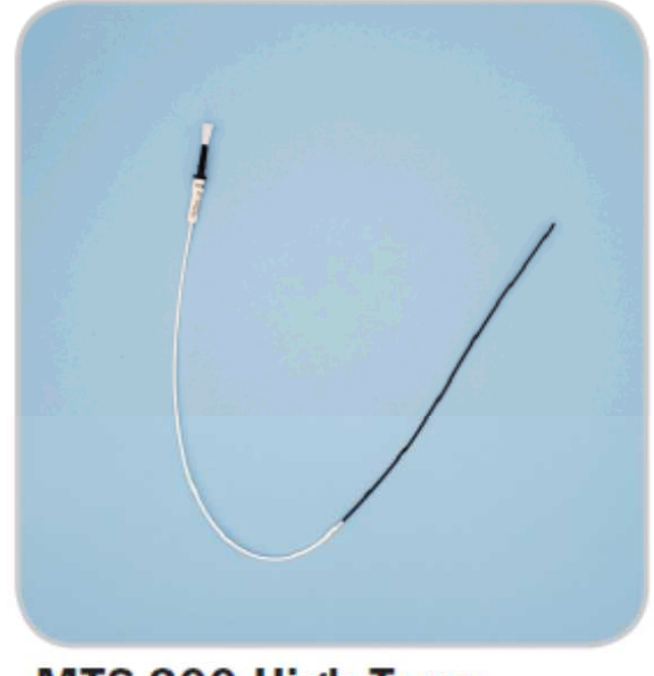

\section{MTS-300 High Temp}

P/N 281461

White probe with black end

Temperatures $>250^{\circ} \mathrm{C}$

MARS 6 only

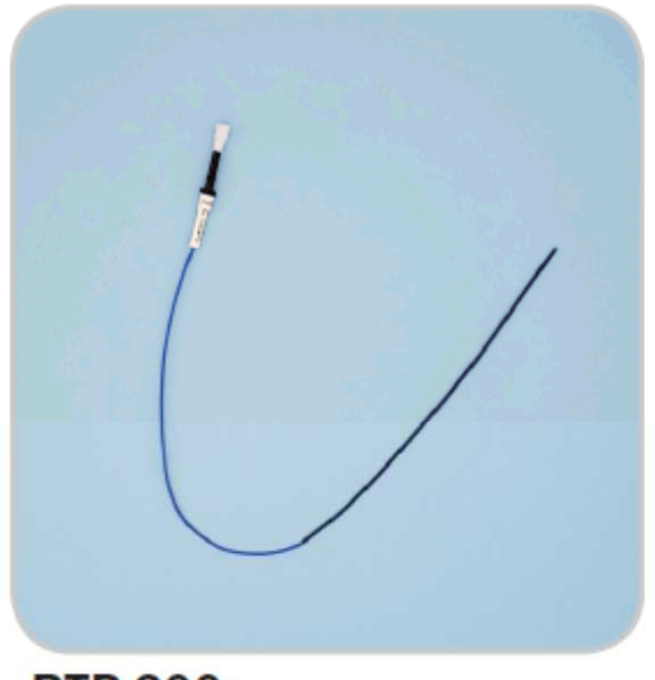

RTP-300+

$\mathrm{P} / \mathrm{N} 314305$

Blue probe with blue end

Temperatures $<250^{\circ} \mathrm{C}$

MARS 5 only

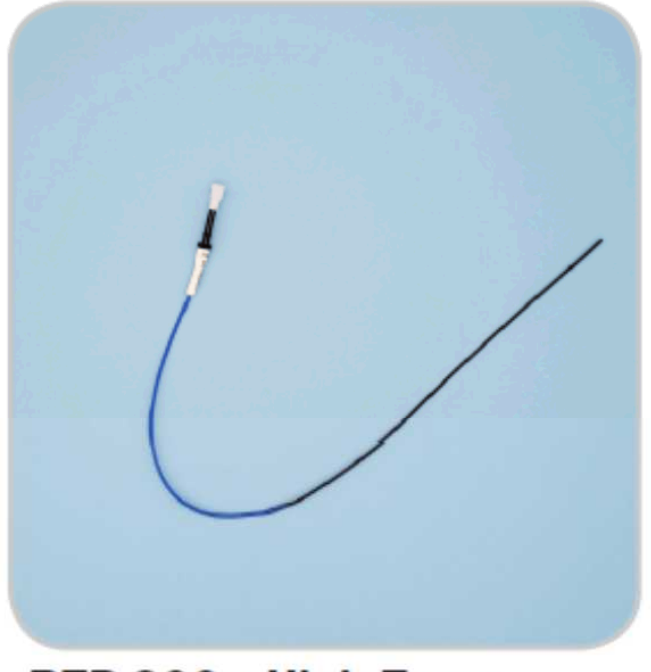

RTP-300+ High Temp

$\mathrm{P} / \mathrm{N} 314306$

Blue probe with black end

Temperatures $>250^{\circ} \mathrm{C}$

MARS 5 only

${ }^{\star}$ RTP-300+ probes are not compatible with the MARS 6 unit as they are shorter than the MTS-300 probe and will not reach the bottom of the thermowell in the control vessel if used in the MARS 6 . 


\section{Run Time Report From microSD Card}

- microSD card removed from the microwave and sent to CEM.

- Method running during the last run was "Mann Soil". A 60-minute run.

- Power $=1800 \mathrm{~W}$

- Ramp over 30 minutes to $170{ }^{\circ} \mathrm{C}$.

- Hold at $170{ }^{\circ} \mathrm{C}$ for 30 minutes.

- Software version 1.26. Latest MARS 6 software is version 1.51

- Thermocouple GF = 448-9482, Probe S/N TJ5009N, MTS-300 MARS 6 probe.

- Power/Temp./Time data from the final run was compared to previous Mann Soil runs that were successfully completed.

- Power/Temp. ramp consistently for $\sim 22$ minutes. At that $22 \mathrm{~min}$. the temperature rise slows significantly and levels at $\sim 141^{\circ} \mathrm{C}$. In response to the temperature lag the microwave went to full power $1800 \mathrm{~W}$ for 14.5 minutes, but the temperature only went to $\sim 149{ }^{\circ} \mathrm{C}$. The run was aborted at 40.1 minutes by the ReactiGuard.

- CEM concludes that continued and prolonged application of $1800 \mathrm{~W}$ caused for overheating and subsequent fire. 


\section{Runtime Data}
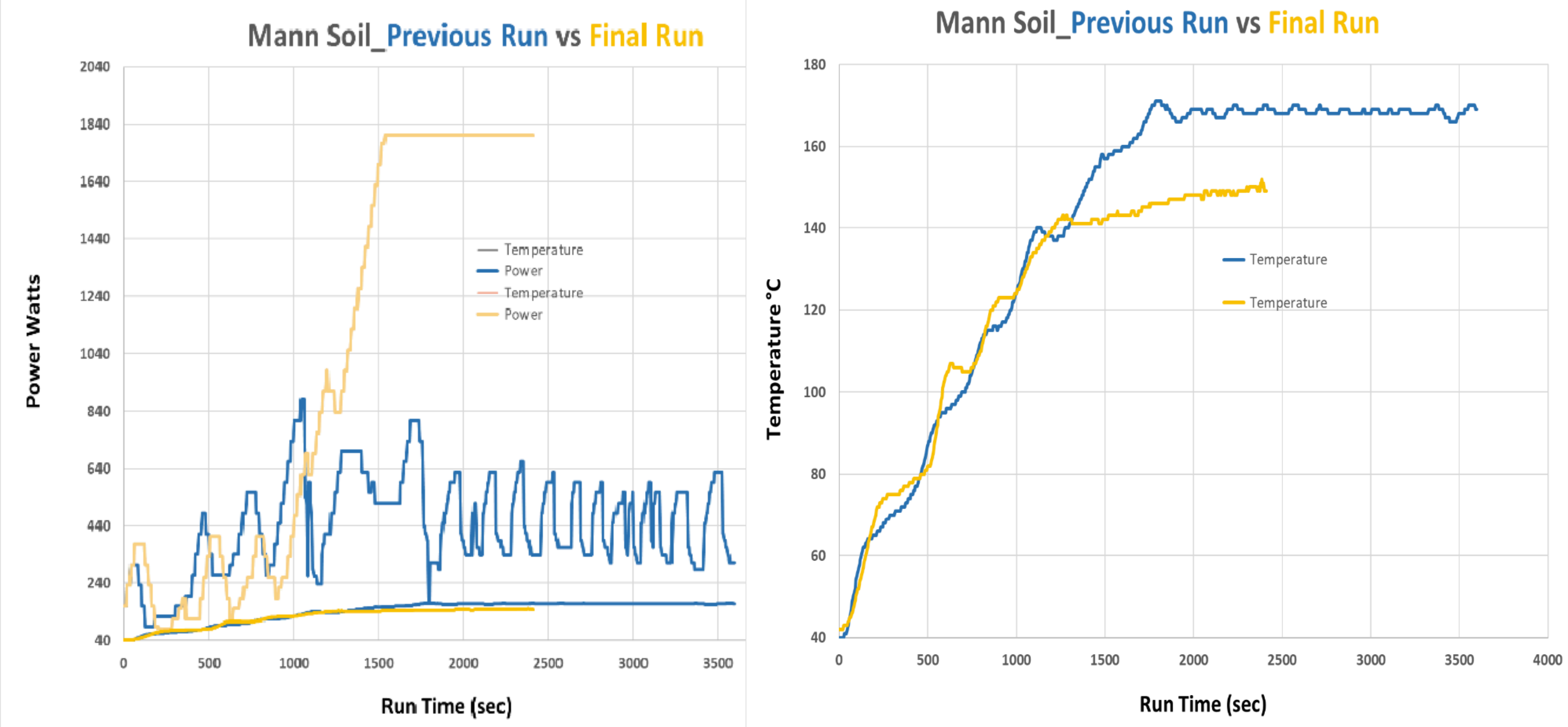


\section{Contributing Factors}

WARNING

- Blue Mars 5 thermocouple probe (16") used in a Mars 6 device. Incorrect S/N and GF.

- Unattended run. Acid fume sensor (optional, but not purchased) on exhaust line not used.

\section{Additional Information: HPI}

- Mars 5 thermocouple kept with Mars 6 spare probes and Mars 6 device.

- Mars 5 thermocouple fits into the Mars 6 connector and will not ERROR OUT.

- Thermocouple product boxes and labeling are similar. Probes not returned to their original boxes.

- At least 3 different colored warning labels containing 4 different warnings in probe boxes.

\section{WARNING}

This WHITE MTS-300 Probe is designed for the MARS 6 Digestion System, and SHOULD NOT be used in a MARS 5.

- A 100-page user manual that mentions nothing about Mars 5/Mars 6 part incompatibility.

- No known product recalls or other warnings for incompatible probes. 


\section{Lessons..........Learned?}

- CEM database showed the microwave was owned by a company located in North Carolina. INL Procurement using $3^{\text {rd }}$ party vendors, who gets the updates?

- Cognizants listed in the CEM database for this instrument were INL staff present at the time of installation.

- User Manual revisions. Critical software revisions. Research staff not notified of updates.

- Storage of incompatible parts with compatible parts.

- Safety equipment listed as "optional" by the manufacturer. Equipment not purchased.

- New probe (safety-critical equipment) installed in a device w/o consulting manufacturer's operating instructions or investigating specifics regarding the thermocouple.

- Incorrect serial\# and Gauge Factor programmed into the instrument.

- No evidence of routine maintenance of the torque wrench.

- Over-reliance on equipment, the manufacturer, on past successful runs, and on personal knowledge/skills.

- Sign blindness. Bright orange WARNING label in the probe box.

- Conditioning/culture that enables us to ignore instructions and warning labels. 